S. Shimomura

Nagoya Math. J.

Vol. 166 (2002), 55-82

\title{
OSCILLATION RESULTS FOR $n$-TH ORDER LINEAR DIFFERENTIAL EQUATIONS WITH MEROMORPHIC PERIODIC COEFFICIENTS
}

\author{
SHUN SHIMOMURA
}

\begin{abstract}
Consider $n$-th order linear differential equations with meromorphic periodic coefficients of the form $w^{(n)}+R_{n-1}\left(e^{z}\right) w^{(n-1)}+\cdots+R_{1}\left(e^{z}\right) w^{\prime}+$ $R_{0}\left(e^{z}\right) w=0, n \geq 2$, where $R_{\nu}(t)(0 \leq \nu \leq n-1)$ are rational functions of $t$. Under certain assumptions, we prove oscillation theorems concerning meromorphic solutions, which contain necessary conditions for the existence of a meromorphic solution with finite exponent of convergence of the zero-sequence. We also discuss meromorphic or entire solutions whose zero-sequences have an infinite exponent of convergence, and give a new zero-density estimate for such solutions.
\end{abstract}

\section{$\S 1$. Introduction}

Consider equations of the form

$$
w^{(n)}+A_{n-1}\left(e^{z}\right) w^{(n-1)}+\cdots+A_{1}\left(e^{z}\right) w^{\prime}+A_{0}\left(e^{z}\right) w=0, \quad n \geq 2
$$

$\left({ }^{\prime}=d / d z\right)$, where $A_{\nu}(t)(0 \leq \nu \leq n-1)$ are rational functions of $t$ admitting poles at most at $t=0, \infty$ only. The coefficients of (1.1) are entire periodic functions, and every solution is entire. In the case where $n=2$, the zero distribution of solutions was first examined by [8]. Studies concerning (1.1) have been carried on by several authors, and various oscillation theorems have been obtained ([2], [4], [12], [14], [15], [16]).

In this paper we extend such results to meromorphic solutions of linear equations with meromorphic periodic coefficients. Some of our results, even in the case restricted to entire solutions, are also improvements of previously known ones concerning equations with entire periodic coefficients. We treat $n$-th order linear differential equations of the form

$$
w^{(n)}+R_{n-1}\left(e^{z}\right) w^{(n-1)}+\cdots+R_{1}\left(e^{z}\right) w^{\prime}+R_{0}\left(e^{z}\right) w=0, \quad n \geq 2 .
$$

Received October 18, 1999.

2000 Mathematics Subject Classification: 34M10, 34M05, 34M30, 30D35. 
Here $R_{\nu}(t)(0 \leq \nu \leq n-1)$ are rational functions of $t$ which may admit poles other than $t=0$ or $\infty$, and hence the coefficients $R_{\nu}\left(e^{z}\right)$ are meromorphic on $\mathbf{C}$. Throughout this paper we suppose the following conditions on $(\mathrm{E})$ :

(a) around $t=\infty$,

$$
\begin{aligned}
& R_{0}(t)=t^{q} \sum_{k=0}^{\infty} a_{k} t^{-k}, \quad q \in \mathbf{N}, a_{0} \neq 0, \\
& R_{\nu}(t)=t^{q} \sum_{k=0}^{\infty} a_{\nu, k} t^{-k} \text { or } \equiv 0, \quad 1 \leq \nu \leq n-1, \\
& q_{\nu} \in \mathbf{Z}, \quad a_{\nu, 0} \neq 0,
\end{aligned}
$$

where

$$
q_{\nu}<q(n-1-\nu) / n \quad \text { for } 1 \leq \nu \leq n-2, \quad q_{n-1} \leq 0
$$

(b) around $t=0$,

$$
\begin{aligned}
& R_{0}(t)=t^{-p} \sum_{k=0}^{\infty} b_{k} t^{k}, \quad p \in \mathbf{Z}, \quad b_{0} \neq 0, \\
& R_{\nu}(t)=t^{-p_{\nu}} \sum_{k=0}^{\infty} b_{\nu, k} t^{k} \quad \text { or } \equiv 0, \quad 1 \leq \nu \leq n-1, \\
& p_{\nu} \in \mathbf{Z}, \quad b_{\nu, 0} \neq 0,
\end{aligned}
$$

where

$$
\begin{aligned}
& p_{\nu}<p(n-1-\nu) / n \quad \text { for } 1 \leq \nu \leq n-2, \quad p_{n-1} \leq 0, \quad \text { if } p \geq 1 \text {, } \\
& p_{\nu} \leq 0 \quad \text { for } 1 \leq \nu \leq n-1, \quad \text { if } p \leq 0 \text {; }
\end{aligned}
$$

(c) equation (E) possesses at least one solution which is nontrivial and meromorphic on the whole complex z-plane $\mathbf{C}$.

We put

$$
\mathcal{P}=\bigcup_{\nu=0}^{n-1} \mathcal{P}_{\nu} \subset \mathbf{C}-\{0\}
$$

where each $\mathcal{P}_{\nu}$ is the set of all the distinct poles of $R_{\nu}(t)$ other than $t=0$ or $\infty$. Clearly $\mathcal{P}$ is a finite set. If $(\mathrm{E})$ possesses a meromorphic solution 
with poles, then $\mathcal{P}$ is not empty. By the change of the variable $t=e^{z},(\mathrm{E})$ is taken into the equation

(aE) $\vartheta^{n} w+R_{n-1}(t) \vartheta^{n-1} w+\cdots+R_{1}(t) \vartheta w+R_{0}(t) w=0, \quad \vartheta=t(d / d t)$,

which is called the associate equation of $(\mathrm{E})$. For an arbitrary solution $\phi(z)$ of $(\mathrm{E})$, there exists a solution $\Phi(t)$ of (aE) such that $\phi(z)=\Phi\left(e^{z}\right)$ at least around a point $z=z_{0}$ at which $\phi(z)$ is analytic. Then, $\Phi(t)$ is continued meromorphically to $\mathcal{R}$, if and only if $\phi(z)$ is meromorphic on $\mathbf{C}$. Here $\mathcal{R}$ denotes the universal covering of $\mathbf{C}-\{0\}$, namely the Riemann surface of $\log t$. In general, solutions of equations of the form (aE) have a branch point at $t=\xi \in \mathcal{P}$. In the case which we are going to treat, the coefficients of (aE) need to satisfy suitable conditions under which (aE) possesses a nontrivial solution meromorphic on $\mathcal{R}$. For example, if every $\xi \in \mathcal{P}$ is an apparent singular point, namely a regular singular point at which all the characteristic exponents are integers and the series expansion of every solution does not contain a logarithmic term, then every solution of (aE) is meromorphic on $\mathcal{R}$. Such conditions for $n=2$ are found in [9], [21, Chapter 6], [22]; see also examples in Section 3.1.2.

Our main results and their corollaries are stated in Sections 2 and 3. Theorem 2.1 is an extension of oscillation results for the entire periodic coefficients cases ([8, Theorem 1], [12, Theorem 2]), which gives necessary conditions for the existence of a meromorphic solution of (E) satisfying $\lambda(\phi)<+\infty$. Here $\lambda(f)$ denotes the exponent of convergence of the zerosequence of a meromorphic function $f$, namely

$$
\lambda(f)=\limsup _{r \rightarrow \infty} \frac{\log N(r, 1 / f)}{\log r},
$$

in which $N(r, g)$ denotes the counting function (see [18], [20], [21]). Theorem 2.3 gives a zero-density estimate for every meromorphic solution of (E) satisfying $\lambda(\phi)=+\infty$. For (1.1) with entire periodic coefficients, it is known that a result corresponding to Theorem 2.1 is also valid under the condition $\log N(r, 1 / w)=o(r)$ instead of $\lambda(w)<+\infty([2],[4],[12])$. Theorem 2.3 enables us to replace this by a weaker condition of the form $\liminf _{r \rightarrow \infty} r^{-1} \log N(r, 1 / w)<C_{0}$ for some $C_{0}>0$ (Remark 2.3). Furthermore, combining this theorem with Corollary 3.3 which follows from Theorem 2.1, we estimate the zero-density of solutions of the Hill equation (Proposition 3.4). Theorem 2.2 or Corollary 3.5 contains the affirmative 
answer to the conjecture by Chiang and Wang [15] that every nontrivial solution of

$$
w^{(n)}+\left(e^{z}+K_{0}\right) w=0, \quad n \geq 3, \quad K_{0} \in \mathbf{C}
$$

satisfies $\lambda(\phi)=+\infty$ (Section 3.2). For (E) with entire periodic coefficients, Theorem 2.4 gives a sufficient condition under which arbitrary linearly independent solutions $\chi_{0}(z), \chi_{1}(z), \ldots, \chi_{n-1}(z)$ satisfy $\max \left\{\lambda\left(\chi_{0}\right), \ldots, \lambda\left(\chi_{n-1}\right)\right\}$ $=+\infty$; it is an extension of $[7$, Section 3 , Fact (B)] (see also [10, Theorem 1], [11], [13, Theorem 4], [23]). In the proofs of these results, our main idea is to examine the asymptotic behaviour of solutions of (aE) near the singular points $t=\infty$ and $t=0$. The asymptotic integration has been used in the study of the zero distribution of solutions of linear equations ([5], [6], [17], [19]). In Section 4, we give asymptotic solutions of (aE) and sectorial domains in which the expressions of them are valid. In Section 5, we define a zero-ample solution at $t=\infty$ (or at $t=0$ ) of $(\mathrm{aE})$, and show that it admits infinitely many zeros in some sectorial domain. Furthermore we give a characterisation of a solution which is not zero-ample. In Section 6, we prove Theorems 2.1 and 2.3. In the proof of Theorem 2.3, in addition to the zero-density estimate in Section 5, we employ the Wiman-Valiron theory. In Sections 7 and 8, observing the relation between solutions of (aE) near $t=\infty$ and near $t=0$ carefully, we prove Theorems 2.2 and 2.4 .

Throughout this paper, in addition to the standard notation of the Nevanlinna theory such as $T(r, f), N(r, f), \lambda(f)$, we use the notation below:

(1) We write $\varphi(r) \ll \psi(r)$ or $\psi(r) \gg \varphi(r)$ as $r \rightarrow \infty$, if $\varphi(r)=O(\psi(r))$ as $r \rightarrow \infty$.

(2) For a set $A, \sharp A$ denotes the cardinal number of $A$.

(3) For $\sigma \in \mathbf{C}$ and for $m \in \mathbf{Z}-\{0\}, O\left[t^{\sigma}\right]_{1 / m}$ denotes a formal series expressed as $t^{\sigma} \sum_{k \geq 0} c_{k} t^{k / m}\left(c_{k} \in \mathbf{C}\right)$. When $f(t)$ admits a convergent series expression of the form $f(t)=t^{\sigma} \sum_{k \geq 0} c_{k} t^{k / m}$ around $t^{1 / m}=0$, we also write as $f(t)=O\left[t^{\sigma}\right]_{1 / m}$.

\section{§. Main theorems}

We define $\alpha_{k}(k \geq 0)$ by

$$
\left[-t^{q} \sum_{0 \leq k \leq q / n} a_{k} t^{-k}\right]^{1 / n}=t^{q / n} \sum_{k \geq 0} \alpha_{k} t^{-k}, \quad \alpha_{0}=\left(-a_{0}\right)^{1 / n},
$$


near $t=\infty$. When $p \in \mathbf{N}$, we define $\beta_{k}(k \geq 0)$ by

$$
\left[(-1)^{n+1} t^{-p} \sum_{0 \leq k \leq p / n} b_{k} t^{k}\right]^{1 / n}=t^{-p / n} \sum_{k \geq 0} \beta_{k} t^{k}, \quad \beta_{0}=-\left(-b_{0}\right)^{1 / n}
$$

near $t=0$. Here $a_{k}, b_{k}(k \geq 0)$ are the coefficients of (1.2) and (1.5). When $q / n \in \mathbf{N}, t^{q / n} \sum_{0 \leq k \leq q / n} \alpha_{k} t^{-k}$ is the approximate $n$-th root of [1], [7]. Put

$$
\begin{aligned}
& V_{\infty}(t)=t^{q / n} \sum_{0 \leq k<q / n} \frac{\alpha_{k}}{q / n-k} t^{-k}, \\
& V_{0}(t)=t^{-p / n} \sum_{0 \leq k<p / n} \frac{\beta_{k}}{p / n-k} t^{k} \quad(p \in \mathbf{N}) .
\end{aligned}
$$

If $p \leq 0$, then we put $V_{0}(t) \equiv 0$.

THEOREM 2.1. Suppose that (E) possesses a meromorphic solution $w=\phi(z)(\not \equiv 0)$ satisfying $\lambda(\phi)<+\infty$. Then $\phi(z)$ is expressible in the form

$$
\begin{aligned}
& \phi(z)=\Phi\left(e^{z}\right) \\
& \Phi(t)=\left(\prod_{\xi \in \mathcal{P}}(t-\xi)^{-\iota(\xi)}\right) P\left(t^{1 / n}\right) t^{\kappa} \exp \left(\omega_{\infty} V_{\infty}(t)+\omega_{0} V_{0}(t)\right),
\end{aligned}
$$

and one of the following must hold:

(i) $q / n \notin \mathbf{N}, p / n \notin \mathbf{N}, p \geq 1$, and

$$
n I_{\phi}(\mathcal{P})-(n-1)(q+p) / 2-\left(R_{n-1}(\infty)-R_{n-1}(0)\right) \in \mathbf{N} \cup\{0\} ;
$$

(ii) $q / n \in \mathbf{N}, p / n \in \mathbf{N}$, and

$$
\begin{aligned}
I_{\phi}(\mathcal{P})-(n-1)(q+p) /(2 n)-\left(R_{n-1}(\infty)\right. & \left.-R_{n-1}(0)\right) / n \\
& +\omega_{\infty} \alpha_{q / n}+\omega_{0} \beta_{p / n} \in \mathbf{N} \cup\{0\}
\end{aligned}
$$

(iii) $q / n \notin \mathbf{N}, p \leq 0$, and, for some $m \in \mathbf{Z}$ satisfying $m \leq n I_{\phi}(\mathcal{P})$,

$$
\rho_{m}=\left(2 m-(n-1) q-2 R_{n-1}(\infty)\right) /(2 n)
$$

is a root of the equation

$$
\rho^{n}+\sum_{\nu=0}^{n-1} R_{\nu}(0) \rho^{\nu}=0
$$


(iv) $q / n \in \mathbf{N}, p \leq 0$, and, for some $m \in \mathbf{Z}$ satisfying $m \leq I_{\phi}(\mathcal{P})$,

$$
\tilde{\rho}_{m}=m-(n-1) q /(2 n)-R_{n-1}(\infty) / n+\omega_{\infty} \alpha_{q / n}
$$

is a root of (2.4).

Here,

(a) $\iota(\xi) \in\{0\} \cup \mathbf{N}, I_{\phi}(\mathcal{P})=\sum_{\xi \in \mathcal{P}} \iota(\xi)$;

(b) $\left(\omega_{\infty}, \omega_{0}\right)$ is some pair of $n$-th roots of 1 ;

(c) $\kappa$ is a constant given by

$$
\kappa= \begin{cases}(n-1) p /(2 n)-R_{n-1}(0) / n & \text { in case (i), } \\ (n-1) p /(2 n)-R_{n-1}(0) / n-\omega_{0} \beta_{p / n} & \text { in case (ii), } \\ \rho_{m} & \text { in case (iii), } \\ \tilde{\rho}_{m} & \text { in case (iv); }\end{cases}
$$

(d) $P(\tau)$ is a polynomial in $\tau$ which satisfies $P(0) \neq 0$ and is not divisible by $\tau^{n}-\xi$ for every $\xi \in \mathcal{P}$ satisfying $\iota(\xi) \in \mathbf{N}$, and in particular, when $q / n \in \mathbf{N}, P\left(t^{1 / n}\right)$ is a polynomial in $t$ such that $P\left(\xi^{1 / n}\right) \neq 0$ for every $\xi \in \mathcal{P}$ satisfying $\iota(\xi) \in \mathbf{N}$.

Remark 2.1. In the theorem above, for each solution $\phi(z)$ such that $\lambda(\phi)<+\infty$, the integer $I_{\phi}(\mathcal{P})$ is uniquely determined. If $\mathcal{P}=\emptyset$, then every solution is entire, and hence $I_{\phi}(\mathcal{P})=0$. There exists a case where $\mathcal{P} \neq \emptyset$ and every solution is entire (see Section 3.1.2).

Remark 2.2. When $\mathcal{P}=\emptyset$, by (1.4) and (1.7) (or (1.8)), we have $R_{n-1}(t) \equiv C \in \mathbf{C}$. Then, by the transformation $w=e^{-C z / n} v$, our problem is reduced to one concerning $(\mathrm{E})$ with $R_{n-1}(t) \equiv 0$.

In the special case where $q / n \notin \mathbf{N}, p \leq 0$, we have the following:

Theorem 2.2. Suppose that $q / n \notin \mathbf{N}$ and that $p \leq 0$. Put $n=n_{0} d_{0}$, $q=q_{0} d_{0}$, where $d_{0}$ is the greatest common divisor of $n$ and $q$. If there exists a meromorphic solution $w=\phi(z)(\not \equiv 0)$ of $(\mathrm{E})$ satisfying $\lambda(\phi)<+\infty$, then

(1) $\phi_{j}(z)=\phi(z+2 j \pi i)\left(j=0,1, \ldots, n_{0}-1\right)$ are linearly independent solutions of $(\mathrm{E})$ satisfying $\lambda\left(\phi_{j}\right)<+\infty$;

(2) the equation

$$
\rho^{n}+\sum_{\nu=0}^{n-1} n_{0}^{n-\nu} R_{\nu}(0) \rho^{\nu}=0
$$


admits $n_{0}$ distinct roots expressed as $-(n-1) q_{0} / 2-R_{n-1}(\infty) / d_{0}+m_{j}$ with $m_{j} \in \mathbf{Z}\left(j=0,1, \ldots, n_{0}-1\right)$ satisfying $m_{0} \leq n_{0} I_{\phi}(\mathcal{P}), m_{0}<m_{1}<\cdots<$ $m_{n_{0}-1}$.

For the zero-density of solutions, we have the following:

THEOREM 2.3. Let $\phi(z)$ be an arbitrary meromorphic solution of $(\mathrm{E})$ satisfying $\lambda(\phi)=+\infty$. Then we have

$$
\log N(r, 1 / \phi)=O(r)
$$

and

$$
\log N(r, 1 / \phi) \geq\left(m_{0}(p, q) / n\right) r+O(\log r)
$$

as $r \rightarrow \infty$, where

$$
m_{0}(p, q)= \begin{cases}\min \{p, q\} & \text { if } p \geq 1 \\ q & \text { if } p \leq 0\end{cases}
$$

Remark 2.3. This theorem implies that the condition $\lambda(\phi)<+\infty$ of Theorem 2.1 or 2.2 can be replaced by

$$
\liminf _{r \rightarrow \infty} r^{-1} \log N(r, 1 / \phi)<m_{0}(p, q) / n
$$

THEOREM 2.4. Suppose that $\mathcal{P}=\emptyset, R_{n-1}(t) \equiv 0$, and that either of the following holds:

(i) $p \geq 1$;

(ii) $p \leq 0, q / n \in \mathbf{N}$, and $\alpha_{q / n}\left(\omega^{j}-\omega^{j^{\prime}}\right) \notin \mathbf{Z}$ for every pair $\left(j, j^{\prime}\right)$ of integers satisfying $0 \leq j<j^{\prime} \leq n-1$, where $\omega=\exp (2 \pi i / n)$.

Then, for arbitrary linearly independent solutions $\chi_{0}(z), \chi_{1}(z), \ldots, \chi_{n-1}(z)$ of $(\mathrm{E})$, we have $\max \left\{\lambda\left(\chi_{0}\right), \lambda\left(\chi_{1}\right), \ldots, \lambda\left(\chi_{n-1}\right)\right\}=+\infty$.

\section{§3. Corollaries and examples}

\subsection{Corollaries of Theorem 2.1}

From Theorem 2.1, we can derive sufficient conditions under which a meromorphic solution of $(\mathrm{E})$ satisfies $\lambda(\phi)=+\infty$. 
Corollary 3.1. Let $\phi(z)=\Phi\left(e^{z}\right)(\not \equiv 0)$ be a meromorphic solution of (E) such that $\Phi(t) \prod_{\xi \in \mathcal{P}}(t-\xi)^{\iota^{*}(\xi)}$ is analytic on $\mathcal{R}$, where $\iota^{*}(\xi) \in \mathbf{N} \cup\{0\}$, $\xi \in \mathcal{P}$. Suppose that, for $I_{\phi}^{*}(\mathcal{P})=\sum_{\xi \in \mathcal{P}} \iota^{*}(\xi)$, one of the following holds:

(i) $q / n \notin \mathbf{N}, p / n \notin \mathbf{N}, p \geq 1$, and

$$
n I_{\phi}^{*}(\mathcal{P})-(n-1)(q+p) / 2-\left(R_{n-1}(\infty)-R_{n-1}(0)\right) \notin \mathbf{N} \cup\{0\} ;
$$

(ii) $q / n \in \mathbf{N}, p / n \in \mathbf{N}$, and, for every $\left(j_{1}, j_{2}\right) \in \mathbf{Z}^{2}$,

$$
\begin{aligned}
& I_{\phi}^{*}(\mathcal{P})-(n-1)(q+p) /(2 n)-\left(R_{n-1}(\infty)-R_{n-1}(0)\right) / n \\
&+\omega^{j_{1}} \alpha_{q / n}+\omega^{j_{2}} \beta_{p / n} \notin \mathbf{N} \cup\{0\},
\end{aligned}
$$

where $\omega=\exp (2 \pi i / n)$.

Then $\lambda(\phi)=+\infty$.

Corollary 3.2. Suppose that $q / n \notin \mathbf{N}, p / n \in \mathbf{N}$, or that $q / n \in \mathbf{N}$, $p / n \notin \mathbf{N}, p \geq 1$. Then every nontrivial meromorphic solution of $(\mathrm{E})$ satisfies $\lambda(\phi)=+\infty$.

Observing that every entire solution $\phi(z)$ of $(\mathrm{E})$ satisfies $I_{\phi}^{*}(\mathcal{P})=0$ in Corollary 3.1, we have the following:

Corollary 3.3. Suppose that $p \geq 1, R_{n-1}(\infty)-R_{n-1}(0) \geq 0$, and that either of the following holds:

(i) $q / n \notin \mathbf{N}$ or $p / n \notin \mathbf{N}$;

(ii) $q / n \in \mathbf{N}, p / n \in \mathbf{N}$, and, for every $\left(j_{1}, j_{2}\right) \in \mathbf{Z}^{2}$,

$$
\begin{aligned}
-(n-1)(q+p) /(2 n)-\left(R_{n-1}(\infty)-\right. & \left.R_{n-1}(0)\right) / n \\
& +\omega^{j_{1}} \alpha_{q / n}+\omega^{j_{2}} \beta_{p / n} \notin \mathbf{N} \cup\{0\} .
\end{aligned}
$$

Then every nontrivial entire solution of (E) satisfies $\lambda(\phi)=+\infty$; under the additional conditions $\mathcal{P}=\emptyset, R_{n-1}(t) \equiv 0$, every nontrivial solution of (E) is entire and satisfies $\lambda(\phi)=+\infty$.

\subsubsection{Hill equation}

Consider the Hill equation

$(\mathrm{HE}) \frac{d^{2} w}{d z^{2}}+\left(\Theta_{0}+2 \Theta_{1} \cos (2 z)+\cdots+2 \Theta_{q} \cos (2 q z)\right) w=0, \quad \Theta_{q} \neq 0$ 
By the change of the variable $s=2 i z,(\mathrm{HE})$ is taken into

$$
\begin{aligned}
& \frac{d^{2} w}{d s^{2}}+R_{0}\left(e^{s}\right) w=0 \\
& R_{0}(t)=-\frac{1}{4}\left(\Theta_{0}+\Theta_{1}\left(t+t^{-1}\right)+\cdots+\Theta_{q}\left(t^{q}+t^{-q}\right)\right) .
\end{aligned}
$$

For every odd integer $q$, it is known that every solution $\phi(z)$ of $(\mathrm{HE})$ satisfies $\lambda(\phi)=+\infty([8])$. When $q$ is even, we put

$$
\left[\frac{t^{q}}{4} \sum_{0 \leq k \leq q / 2} \Theta_{q-k} t^{-k}\right]^{1 / 2}=t^{q / 2} \sum_{k \geq 0} \alpha_{k} t^{-k}, \quad \alpha_{0}=\Theta_{q}^{1 / 2} / 2 .
$$

Note that $\alpha_{q / 2}=\beta_{q / 2}$. For example, if $\Theta_{q-1}=\Theta_{q-2}=\cdots=\Theta_{q-l}=0, l \geq$ $q / 4$, then $\alpha_{q / 2}=\beta_{q / 2}=\Theta_{q / 2} \Theta_{q}^{-1 / 2} / 4$. By Corollary 3.3 and Theorem 2.3, we have the following:

Proposition 3.4. Suppose that (HE) has either of the following properties:

(i) $q$ is odd;

(ii) $q$ is even, and $\pm 2 \alpha_{q / 2}-q / 2 \notin \mathbf{N} \cup\{0\}$.

Then every solution $\phi(z)$ of $(\mathrm{HE})$ satisfies

$$
\log N(r, 1 / \phi) \geq q r+O(\log r), \quad \log N(r, 1 / \phi)=O(r)
$$

as $r \rightarrow \infty$.

3.1.2. Meromorphic coefficients cases

Consider equations of the form

$$
w^{\prime \prime}+R_{1}\left(e^{z}\right) w^{\prime}+R_{0}\left(e^{z}\right) w=0
$$

with

$$
R_{1}(t)=0, \quad R_{0}(t)=\frac{-2}{(t-1)^{2}}+\frac{-3}{t-1}-\frac{1}{2}\left(t^{3}+\frac{1}{t^{3}}\right)
$$

with

$$
R_{1}(t)=-1, \quad R_{0}(t)=-\frac{t}{t-1}-t
$$


and with

$$
R_{1}(t)=\frac{-t}{t-1}, \quad R_{0}(t)=2-t^{2}-\frac{1}{t} .
$$

The associate equations of $\left(\mathrm{E}_{2}\right)$ with (3.1), (3.2), (3.3) possess linearly independent solutions given by

$$
\begin{array}{lll}
\Phi_{1}^{1}(t)=T^{-1}\left(1+O\left[T^{4}\right]_{1}\right), & \Phi_{1}^{2}(t)=T^{2}\left(1+O[T]_{1}\right), \\
\Phi_{2}^{1}(t)=T\left(1+O[T]_{1}\right), & \Phi_{2}^{2}(t)=1+O[T]_{1}+\Phi_{2}^{1}(t) \log T, \\
\Phi_{3}^{1}(t)=1+O\left[T^{3}\right]_{1}, & \Phi_{3}^{2}(t)=T^{2}\left(1+O[T]_{1}\right)
\end{array}
$$

$(T=t-1)$, respectively, around the regular singular point $t=1$. By (3.4), every solution $\phi(z)(\not \equiv 0)$ of $\left(\mathrm{E}_{2}\right)$ with $(3.1)$ is meromorphic. By Corollary 3.1 with $I_{\phi}^{*}(\mathcal{P})=1$, we have $\lambda(\phi)=+\infty$. Equation $\left(\mathrm{E}_{2}\right)$ with $(3.2)$ possesses a one-parameter family of entire solutions $\left\{\phi_{C}(z)=C \Phi\left(e^{z}\right) \mid C \in\right.$ C . (Note that every solution of the associate equation is analytic around $t=0$.) By Theorem 2.1, (iii), we have $\lambda\left(\phi_{C}\right)=+\infty$ for every $C \in \mathbf{C}-\{0\}$. Although $R_{1}\left(e^{z}\right)$ with $(3.3)$ is meromorphic, every solution of $\left(\mathrm{E}_{2}\right)$ with (3.3) is entire. By Corollary 3.2, it satisfies $\lambda(\phi)=+\infty$.

\subsection{Corollaries of Theorem 2.2}

From Theorem 2.2, we immediately have the following:

COROLlary 3.5. Suppose that $q$ and $n$ are relatively prime, and that $p \leq 0$. If the characteristic equation

$$
\rho^{n}+\sum_{\nu=0}^{n-1} n^{n-\nu} R_{\nu}(0) \rho^{\nu}=0
$$

has a multiple root or has a root $\rho_{*}$ such that $\rho_{*}+(n-1) q / 2+R_{n-1}(\infty) \notin \mathbf{Z}$, then every meromorphic solution $\phi(z)$ of $(\mathrm{E})$ satisfies $\lambda(\phi)=+\infty$.

Corollary 3.6. Under the same supposition as in Corollary 3.5, if (E) possesses a meromorphic solution $\phi(z)(\not \equiv 0)$ such that $\lambda(\phi)<+\infty$, then $\phi_{j}(z)=\phi(z+2 j \pi i)(j=0,1, \ldots, n-1)$ are linearly independent solutions of $(\mathrm{E})$ satisfying $\lambda\left(\phi_{j}\right)<+\infty$.

Consider an equation of the form

$$
\begin{aligned}
& w^{(n)}+K_{1} w^{\prime}+R_{0}\left(e^{z}\right) w=0, \quad n \geq 3, \\
& R_{0}(t)=L_{q} t^{q}+\cdots+L_{1} t+L_{0}, \quad L_{q} \neq 0, \quad L_{k} \in \mathbf{C}(0 \leq k \leq q), \\
& K_{1} \in \mathbf{C}-\{x \mid x<0\},
\end{aligned}
$$


where $n$ and $q$ are relatively prime. Equation (3.7) is written in the form

$$
\rho^{n}+n^{n-1} K_{1} \rho+n^{n} L_{0}=0
$$

Since $n \geq 3$, (3.9) has a root $\rho=\rho_{*}$ such that $\operatorname{Im} \rho_{*} \neq 0$, or has a multiple root $\rho=0$. Hence every solution of (3.8) satisfies $\lambda(\phi)=+\infty$. This result is an extension of [15, Theorem 3.2].

In the case where $n=2, R_{1}(t) \equiv 0, R_{0}(t)=t+K_{0}$ or in the case where $n=3, R_{2}(t) \equiv 0, R_{1}(t) \equiv K_{1}, R_{0}(t)=t+K_{0}$, a result corresponding to Corollary 3.6 is known ([10], [15, Theorem 3.1]). For example, as is shown in [15], when $K_{1}=-7 / 9$, the equation

$$
w^{(3)}+K_{1} w^{\prime}+\left(e^{z}-2 / 9\right) w=0, \quad K_{1} \in \mathbf{C}
$$

has the linearly independent solutions

$$
\begin{aligned}
& \phi_{0}(z)=\left(1+(3 / 2) e^{z / 3}\right) \exp \left(-3 e^{z / 3}-(2 / 3) z\right), \\
& \phi_{1}(z)=\phi_{0}(z+2 \pi i), \quad \phi_{2}(z)=\phi_{0}(z+4 \pi i)
\end{aligned}
$$

satisfying $\lambda\left(\phi_{j}\right)<+\infty(j=0,1,2)$. The characteristic equation corresponding to (3.10) is given by

$$
\rho^{3}+9 K_{1} \rho-6=0 .
$$

When $K_{1}=-7 / 9$, (3.11) has the roots $-2,-1,3 \in \mathbf{Z}$. For every $K_{1} \in$ $\mathbf{C}-\{-7 / 9\},(3.11)$ has a root $\rho=\rho_{*} \notin \mathbf{Z}$. Hence by Corollary 3.5, every solution of (3.10) with $K_{1} \neq-7 / 9$ satisfies $\lambda(\phi)=+\infty$.

\section{§4. Asymptotic solutions of $(\mathrm{aE})$}

\subsection{Propositions}

Formal solutions of (aE) are given by the following:

Proposition 4.1. Near $t=\infty$, equation (aE) possesses formal solutions of the form

$$
\begin{aligned}
& W_{j}(t)=Y_{j}(t) \exp \left(\omega^{j} V_{\infty}(t)+\kappa_{j} \log t\right), \quad j=0,1, \ldots, n-1, \\
& \omega=\exp (2 \pi i / n), \\
& \kappa_{j}= \begin{cases}-(n-1) q /(2 n)-R_{n-1}(\infty) / n & \text { if } q / n \notin \mathbf{N}, \\
-(n-1) q /(2 n)-R_{n-1}(\infty) / n+\omega^{j} \alpha_{q / n} & \text { if } q / n \in \mathbf{N} .\end{cases}
\end{aligned}
$$


Here $V_{\infty}(t)$ is the function given by $(2.1)$ and $Y_{j}(t)(0 \leq j \leq n-1)$ are formal power series of the form

$$
Y_{j}(t)=\sum_{h \geq 0} c_{j}(h) t^{-h / n}, \quad c_{j}(0)=1 .
$$

In particular, when $q / n \in \mathbf{N}$,

$$
Y_{j}(t)=\sum_{h \geq 0} c_{j}(n h) t^{-h} .
$$

Let $M_{\infty}$ be a sufficiently large positive constant and $\delta$ a sufficiently small positive constant. For each $\mu \in \mathbf{Z}$, in the universal covering $\mathcal{R}$ of $\mathbf{C}-\{0\}$, we define the sector $S_{\mu}$ by

$$
S_{\mu}=\left\{t \in \mathcal{R}|\mu \pi-\delta<(q / n) \arg t<(\mu+1) \pi,| t \mid>M_{\infty}\right\} .
$$

Then $\bigcup_{\mu \in \mathbf{Z}} S_{\mu}=\mathcal{R}_{\infty}=\left\{t \in \mathcal{R}|| t \mid>M_{\infty}\right\}$.

Proposition 4.2. For each sector $S_{\mu}(\mu \in \mathbf{Z})$, equation (aE) possesses linearly independent solutions $\varphi_{\mu, 0}(t), \ldots, \varphi_{\mu, n-1}(t)$ which admit the asymptotic representations

$$
\varphi_{\mu, j}(t) \sim W_{j}(t), \quad j=0,1, \ldots, n-1,
$$

as $t \rightarrow \infty$ through the sector $S_{\mu}$. Furthermore these solutions are uniquely determined by (4.1).

When $p \in \mathbf{N}$, we also have the following:

Proposition 4.3. Suppose that $p \in \mathbf{N}$. Near $t=0$, equation (aE) possesses formal solutions of the form

$$
\begin{aligned}
& W_{j}^{(0)}(t)=Y_{j}^{(0)}(t) \exp \left(\omega^{j} V_{0}(t)-\kappa_{j}^{0} \log t\right), \quad j=0,1, \ldots, n-1, \\
& \kappa_{j}^{0}= \begin{cases}-(n-1) p /(2 n)+R_{n-1}(0) / n & \text { if } p / n \notin \mathbf{N}, \\
-(n-1) p /(2 n)+R_{n-1}(0) / n+\omega^{j} \beta_{p / n} & \text { if } p / n \in \mathbf{N} .\end{cases}
\end{aligned}
$$

Here $V_{0}(t)$ is the function given by $(2.2)$ and $Y_{j}^{(0)}(t)(0 \leq j \leq n-1)$ are formal power series of the form

$$
Y_{j}^{(0)}(t)=\sum_{h \geq 0} c_{j}^{0}(h) t^{h / n}, \quad c_{j}^{0}(0)=1 .
$$


In particular, when $p / n \in \mathbf{N}$,

$$
Y_{j}^{(0)}(t)=\sum_{h \geq 0} c_{j}^{0}(n h) t^{h} .
$$

For each $\mu \in \mathbf{Z}$, denote by $S_{\mu}^{0}$ the sector given by

$$
S_{\mu}^{0}=\left\{t \in \mathcal{R}|\mu \pi<(p / n) \arg t<(\mu+1) \pi+\delta,| t \mid<\varepsilon_{0}\right\},
$$

where $\delta$ and $\varepsilon_{0}$ are sufficiently small positive constants.

Proposition 4.4. Under the supposition $p \in \mathbf{N}$, for each sector $S_{\mu}^{0}$ $(\mu \in \mathbf{Z})$, equation (aE) possesses linearly independent solutions $\varphi_{\mu, 0}^{(0)}(t), \ldots$, $\varphi_{\mu, n-1}^{(0)}(t)$ which admit the asymptotic representations

$$
\varphi_{\mu, j}^{(0)}(t) \sim W_{j}^{(0)}(t), \quad j=0,1, \ldots, n-1,
$$

as $t \rightarrow 0$ through the sector $S_{\mu}^{0}$. Furthermore these solutions are uniquely determined by (4.2).

Propositions 4.3 and 4.4 are obtained from Propositions 4.1 and 4.2 by putting $t=1 / \tau$ and using (1.5).

\subsection{Proofs of Propositions 4.1 and 4.2}

Let $w$ be an arbitrary solution of (aE). Then the column vector function

$$
\mathbf{w}=D(t)\left(\begin{array}{c}
w \\
\vartheta w \\
\vdots \\
\vartheta^{n-1} w
\end{array}\right), \quad D(t)=\operatorname{diag}\left[1, t^{-q / n}, \ldots, t^{-q(n-1) / n}\right]
$$

satisfies a system of the form

(S)

$$
\begin{aligned}
& \vartheta \mathbf{w}=A(t) \mathbf{w}, \\
& A(t)=D(t) \Xi(t) D(t)^{-1}-D(t) \vartheta\left(D(t)^{-1}\right), \\
& \Xi(t)=\left(\begin{array}{cccccc}
0 & 1 & 0 & \cdots & \cdots & 0 \\
\vdots & 0 & 1 & \ddots & & \vdots \\
\vdots & & \ddots & \ddots & 0 & \vdots \\
\vdots & \vdots & & \ddots & 1 & 0 \\
0 & 0 & \cdots & \cdots & 0 & 1 \\
-R_{0} & -R_{1} & \cdots & \cdots & -R_{n-2} & -R_{n-1}
\end{array}\right) .
\end{aligned}
$$


Observing (1.4), we can verify that

$$
\begin{aligned}
& A(t)=t^{q / n} \sum_{k \geq 0} A_{k} t^{-k}+\sum_{\nu=0}^{n-2} t^{-q \nu / n} \sum_{k \geq-q \nu / n} A_{k}^{(\nu)} t^{-k}-D_{0}, \\
& A_{0}=\left(\begin{array}{ccccc}
0 & 1 & 0 & \cdots & 0 \\
\vdots & 0 & 1 & \ddots & \vdots \\
\vdots & \vdots & \ddots & \ddots & 0 \\
0 & 0 & \cdots & 0 & 1 \\
-a_{0} & 0 & \cdots & \cdots & 0
\end{array}\right), \quad D_{0}=(q / n) \operatorname{diag}[0,1, \ldots, n-1] .
\end{aligned}
$$

Note that $A_{0}$ has the distinct eigenvalues $\omega^{j}\left(-a_{0}\right)^{1 / n}=\omega^{j} \alpha_{0}(j=0,1, \ldots$, $n-1)$. When $q / n \in \mathbf{N}$, system (S) admits a formal fundamental matrix solution of the form

$$
U(t) \exp \left(t^{q / n} \sum_{0 \leq k<q / n} \Delta_{k} t^{-k}+\Delta_{*} \log t\right), \quad U(t)=\sum_{k \geq 0} U_{k} t^{-k}
$$

Here $U_{k}(k \geq 0)$ are $n$ by $n$ matrices, and $\Delta_{k}(0 \leq k<q / n), \Delta_{*}$ are diagonal matrices; in particular $\Delta_{0}=\left(\alpha_{0} /(q / n)\right) \operatorname{diag}\left[1, \omega, \ldots, \omega^{n-1}\right]$, and $U_{0} \in G L(n, \mathbf{C})$ satisfies $U_{0}^{-1} A_{0} U_{0}=\alpha_{0} \operatorname{diag}\left[1, \omega, \ldots, \omega^{n-1}\right]$ (see $[26$, Sections 10,11$])$. Hence equation (aE) has formal solutions of the form

$$
\begin{aligned}
& \tilde{W}_{j}(t)=\tilde{Y}_{j}(t) \exp \left(t^{q / n} \sum_{0 \leq k<q / n} \tilde{\alpha}_{j, k} t^{-k}+\tilde{\kappa}_{j} \log t\right) \\
& \tilde{Y}_{j}(t)=\sum_{h \geq 0} \tilde{c}_{j, h} t^{-h}, \quad \tilde{c}_{j, 0}=1, \quad \tilde{\alpha}_{j, 0}=\frac{\omega^{j} \alpha_{0}}{q / n} \quad(0 \leq j \leq n-1) .
\end{aligned}
$$

In the case where $q / n \notin \mathbf{N}$, putting $\tau=t^{1 / n}$ in $(\mathrm{S})$, we have

$$
\tilde{\vartheta} \mathbf{w}=n A\left(\tau^{n}\right) \mathbf{w}, \quad \tilde{\vartheta}=\tau(d / d \tau)
$$

with

$$
n A\left(\tau^{n}\right)=\tau^{q} \sum_{0 \leq k<q / n} n A_{k} \tau^{-n k}+\sum_{k \geq 0} A_{k}^{\prime} \tau^{-k}
$$


From this we obtain formal solutions of (aE) expressed as

$$
\begin{aligned}
& \tilde{\tilde{W}}_{j}(t)=\tilde{\tilde{Y}}_{j}(t) \exp \left(t^{q / n} \sum_{0 \leq k<q / n} \tilde{\tilde{\alpha}}_{j, k} t^{-k}+\tilde{\tilde{\kappa}}_{j} \log t\right), \\
& \tilde{\tilde{Y}}_{j}(t)=\sum_{h \geq 0} \tilde{\tilde{c}}_{j, h} t^{-h / n}, \quad \tilde{\tilde{c}}_{j, 0}=1, \quad \tilde{\tilde{\alpha}}_{j, 0}=\frac{\omega^{j} \alpha_{0}}{q / n} \quad(0 \leq j \leq n-1) .
\end{aligned}
$$

It is known that, for each sector $S_{\mu}$, there exist uniquely determined linearly independent solutions $\varphi_{\mu, 0}(t), \ldots, \varphi_{\mu, n-1}(t)$ of $(\mathrm{aE})$ admitting the asymptotic representations

$$
\varphi_{\mu, j}(t) \sim \tilde{W}_{j}(t) \quad\left(\text { or } \sim \tilde{W}_{j}(t)\right), \quad j=0,1, \ldots, n-1
$$

as $t \rightarrow \infty$ through the sector $S_{\mu}([3$, Theorem A], see also [24], [25]).

By the facts above, it is sufficient to show that (4.3) (or (4.4)) coincides with the formal solution $W_{j}(t)$ of Proposition 4.1 . We write $\tilde{W}_{j}(t)$ in the form

$$
\tilde{W}_{j}(t)=\exp \left(\Omega_{j}(t)\right), \quad \Omega_{j}(t)=t^{q / n} \sum_{0 \leq k<q / n} \tilde{\alpha}_{j, k} t^{-k}+\tilde{\kappa}_{j} \log t+O\left[t^{-1}\right]_{-1} .
$$

By induction on $\nu \in \mathbf{N}$, we can verify that

$$
\vartheta^{\nu}=t^{\nu} \frac{d^{\nu}}{d t^{\nu}}+N_{\nu-1}^{\nu} t^{\nu-1} \frac{d^{\nu-1}}{d t^{\nu-1}}+\cdots+N_{1}^{\nu} t \frac{d}{d t}, \quad N_{\nu-1}^{\nu}=\nu(\nu-1) / 2,
$$

and that

$$
\begin{aligned}
& \left(\exp \left(\Omega_{j}(t)\right)\right)^{(\nu)} / \exp \left(\Omega_{j}(t)\right) \\
& \quad=\Omega_{j}^{\prime}(t)^{\nu}+N_{\nu-1}^{\nu} \Omega_{j}^{\prime}(t)^{\nu-2} \Omega_{j}^{\prime \prime}(t)+O\left[t^{(\nu-2) q / n-\nu}\right]_{-1} .
\end{aligned}
$$

Using (1.4) and observing $q / n \in \mathbf{N}$, we have

$$
\begin{aligned}
& \tilde{W}_{j}(t)^{-1}\left(\vartheta^{n}+R_{n-1}(t) \vartheta^{n-1}+\cdots+R_{1}(t) \vartheta+R_{0}(t)\right) \tilde{W}_{j}(t) \\
& =\tilde{W}_{j}(t)^{-1}\left(\vartheta^{n}+R_{n-1}(t) \vartheta^{n-1}\right) \tilde{W}_{j}(t)+R_{0}(t)+O\left[t^{(n-1) q / n-1}\right]_{-1} \\
& =\left(t \Omega_{j}^{\prime}(t)\right)^{n}+\frac{1}{2} n(n-1)\left(\left(t \Omega_{j}^{\prime}(t)\right)^{n-2}\left(t^{2} \Omega_{j}^{\prime \prime}(t)\right)+\left(t \Omega_{j}^{\prime}(t)\right)^{n-1}\right) \\
& \quad+R_{n-1}(\infty)\left(t \Omega_{j}^{\prime}(t)\right)^{n-1}+R_{0}(t)+O\left[t^{(n-1) q / n-1}\right]_{-1} \\
& \quad 0 .
\end{aligned}
$$


Note that $t \Omega_{j}^{\prime}(t)=\omega^{j} \alpha_{0} t^{q / n}\left(1+O\left[t^{-1}\right]_{-1}\right)$, and that

$$
\begin{aligned}
& \left(t \Omega_{j}^{\prime}(t)\right)^{n-2}\left(t^{2} \Omega_{j}^{\prime \prime}(t)\right)+\left(t \Omega_{j}^{\prime}(t)\right)^{n-1} \\
& \quad=\omega^{-j}(q / n) \alpha_{0}^{n-1} t^{(n-1) q / n}+O\left[t^{(n-1) q / n-1}\right]_{-1} .
\end{aligned}
$$

By the definition of $V_{\infty}(t)$,

$$
-R_{0}(t)=\left(t V_{\infty}^{\prime}(t)\right)^{n}+n \alpha_{0}^{n-1} \alpha_{q / n} t^{(n-1) q / n}+O\left[t^{(n-1) q / n-1}\right]_{-1} .
$$

Substitution of these into (4.5) yields

$$
\begin{aligned}
& \left(t \Omega_{j}^{\prime}(t)\right)^{n}-\left(t V_{\infty}^{\prime}(t)\right)^{n} \\
& -n \omega^{-j}\left(\omega^{j} \alpha_{q / n}-(n-1) q /(2 n)-R_{n-1}(\infty) / n\right) \alpha_{0}^{n-1} t^{(n-1) q / n} \\
& \quad+O\left[t^{(n-1) q / n-1}\right]_{-1}=0
\end{aligned}
$$

from which we obtain

$$
t \Omega_{j}^{\prime}(t)=\omega^{j} t V_{\infty}^{\prime}(t)+\left(\omega^{j} \alpha_{q / n}-(n-1) q /(2 n)-R_{n-1}(\infty) / n\right)+O\left[t^{-1}\right]_{-1} .
$$

This implies that $\tilde{W}_{j}(t)$ coincides with $W_{j}(t)$. In case $q / n \notin \mathbf{N}$, replacing $O\left[t^{-1}\right]_{-1}, O\left[t^{(n-1) q / n-1}\right]_{-1}$ by $O\left[t^{-1 / n}\right]_{-1 / n}, O\left[t^{(n-1) q / n-1 / n}\right]_{-1 / n}$, respectively, in the argument above, and using

$$
\begin{aligned}
& \left(t \Omega_{j}^{\prime}(t)\right)^{n-2}\left(t^{2} \Omega_{j}^{\prime \prime}(t)\right)+\left(t \Omega_{j}^{\prime}(t)\right)^{n-1} \\
& \quad=\omega^{-j}(q / n) \alpha_{0}^{n-1} t^{(n-1) q / n}+O\left[t^{(n-1) q / n-1 / n}\right]_{-1 / n} \\
& -R_{0}(t)=\left(t V_{\infty}^{\prime}(t)\right)^{n}+O\left[t^{(n-1) q / n-1 / n}\right]_{-1 / n}
\end{aligned}
$$

instead of $(4.6),(4.7)$, respectively, we can verify that $\tilde{\tilde{W}}_{j}(t)=W_{j}(t)$. Thus the propositions are proved.

\section{§5. Zero-ample solutions of $(\mathrm{aE})$}

Recall the sector $S_{\mu}$ and the corresponding linearly independent solutions $\varphi_{\mu, 0}(t), \ldots, \varphi_{\mu, n-1}(t)$ of (aE) given by Proposition 4.2 . Let $\chi(t)$ be an arbitrary nontrivial solution of $(\mathrm{aE})$. In each sector $S_{\mu}$, it is uniquely expressed as

$$
\chi(t)=\gamma_{\mu, 0} \varphi_{\mu, 0}(t)+\cdots+\gamma_{\mu, n-1} \varphi_{\mu, n-1}(t), \quad \gamma_{\mu, j} \in \mathbf{C} .
$$

We call $\chi(t)$ a zero-ample solution at $t=\infty$, if, for some $\mu(\in \mathbf{Z})$, there exist at least two distinct indices $j, j^{\prime}\left(0 \leq j<j^{\prime} \leq n-1\right)$ such that $\gamma_{\mu, j} \gamma_{\mu, j^{\prime}} \neq 0$. 
Proposition 5.1. Let $\chi(t)$ be a zero-ample solution at $t=\infty$. Then, for some sector $S_{\mu}$,

$$
\sharp\left\{t \in S_{\mu}\left|\chi(t)=0, M_{\infty}<\right| t \mid<r\right\} \gg r^{q / n}
$$

as $r \rightarrow \infty$.

Proof. There exists a sector $S_{\mu}$ such that expression (5.1) of $\chi(t)$ contains at least two non-vanishing coefficients. Since the opening of $S_{\mu}$ is larger than $n \pi / q$, there exist a pair $\left(j_{1}, j_{2}\right)\left(j_{1} \neq j_{2}\right)$ of indices and the direction $\arg t=\theta_{0}=\theta_{0}\left(j_{1}, j_{2}\right)$ in the interior of $S_{\mu}$ with the properties:

(1) $\gamma_{\mu, j_{1}} \gamma_{\mu, j_{2}} \neq 0$

(2) $\operatorname{Re}\left(\left(\omega^{j_{2}}-\omega^{j_{1}}\right) \alpha_{0} t^{q / n}\right)=0$ on the ray $\arg t=\theta_{0}$;

(3) for every $j$ satisfying $j \neq j_{1}, j_{2}$ and $\gamma_{\mu, j} \neq 0, \operatorname{Re}\left(\left(\omega^{j}-\omega^{j_{1}}\right) \alpha_{0} t^{q / n}\right)<$ 0 on the ray $\arg t=\theta_{0}$.

Then, for a sufficiently small positive constant $\varepsilon$, we have

$$
\begin{aligned}
\chi(t) & =\gamma_{\mu, j_{2}} \varphi_{\mu, j_{1}}(t)\left[\gamma_{\mu, j_{1}} / \gamma_{\mu, j_{2}}+\varphi_{\mu, j_{2}}(t) / \varphi_{\mu, j_{1}}(t)+o(1)\right] \\
= & \gamma_{\mu, j_{2}} \varphi_{\mu, j_{1}}(t)\left[\gamma_{\mu, j_{1}} / \gamma_{\mu, j_{2}}\right. \\
& \left.\quad+\exp \left(\left(\omega^{j_{2}}-\omega^{j_{1}}\right)\left(n \alpha_{0} / q\right) t^{q / n}(1+o(1))\right)+o(1)\right]
\end{aligned}
$$

as $t \rightarrow \infty$ through $S\left(\theta_{0}, \varepsilon\right)=\left\{t \in S_{\mu}|| \arg t-\theta_{0} \mid<\varepsilon\right\}$. This yields

$$
\sharp\left\{t \in S\left(\theta_{0}, \varepsilon\right)\left|\chi(t)=0, M_{\infty}<\right| t \mid<r\right\} \gg r^{q / n},
$$

from which the desired estimate follows.

Proposition 5.2. Suppose that $\psi(t)(\not \equiv 0)$ is not a zero-ample solution at $t=\infty$. Then, in every sector $S_{\mu}$, we have

$$
\psi(t)=\gamma_{0} \varphi_{\mu, j_{*}}(t)
$$

where the constant $\gamma_{0}(\neq 0)$ and the index $j_{*}$ are independent of $\mu$.

Proof. By definition, $\psi(t)$ is expressed as $\psi(t)=\tilde{\gamma}_{\mu, j(\mu)} \varphi_{\mu, j(\mu)}(t)$ $\left(\tilde{\gamma}_{\mu, j(\mu)} \neq 0\right)$ in each $S_{\mu}$. By Proposition 4.2,

$$
\psi(t) \sim \tilde{\gamma}_{\mu, j(\mu)} W_{j(\mu)}(t), \quad \text { and } \quad \psi(t) \sim \tilde{\gamma}_{\mu+1, j(\mu+1)} W_{j(\mu+1)}(t)
$$

as $t \rightarrow \infty$ through $S_{\mu} \cap S_{\mu+1} \neq \emptyset$. Viewing the asymptotic behaviour, we have $j(\mu)=j(\mu+1)$ and $\tilde{\gamma}_{\mu, j(\mu)}=\tilde{\gamma}_{\mu+1, j(\mu+1)}$. Repeating this procedure, we can verify the assertion. 
Proposition 5.3. For each $(\mu, j)(\mu \in \mathbf{Z}, 0 \leq j \leq n-1)$, the solution $\varphi_{\mu, j}(t)$ of Proposition 4.2 is not zero-ample at $t=\infty$, if and only if the formal series $Y_{j}(t)$ is convergent around $t=\infty$.

Proof. If $Y_{j}(t)$ is convergent, then, clearly, $\varphi_{\mu, j}(t)=W_{j}(t)\left(t \in \mathcal{R}_{\infty}\right)$ is not zero-ample. Suppose that $\varphi_{\mu, j}(t)$ is not zero-ample, and that $t \in S_{\mu}$. Note that $e^{2 n \pi i} t \in S_{\mu+2 q}$. By Propositions 5.2 and 4.2,

$$
\varphi_{\mu, j}\left(e^{2 n \pi i} t\right)=\varphi_{\mu+2 q, j}\left(e^{2 n \pi i} t\right) \sim W_{j}\left(e^{2 n \pi i} t\right)=e^{2 n \kappa_{j} \pi i} W_{j}(t)
$$

as $e^{2 n \pi i} t \rightarrow \infty$ through $S_{\mu+2 q}$, namely as $t \rightarrow \infty$ through $S_{\mu}$. On the other hand, by the monodromic property, there exist constants $C_{0}, \ldots, C_{n-1}$ such that

$$
\varphi_{\mu, j}\left(e^{2 n \pi i} t\right)=C_{0} \varphi_{\mu, 0}(t)+\cdots+C_{n-1} \varphi_{\mu, n-1}(t),
$$

and hence, by Proposition 4.2,

$$
\varphi_{\mu, j}\left(e^{2 n \pi i} t\right) \sim C_{0} W_{0}(t)+\cdots+C_{n-1} W_{n-1}(t)
$$

as $t \rightarrow \infty$ through $S_{\mu}$. Since the opening of $S_{\mu}$ is larger than $n \pi / q$, from (5.2) and (5.4) it follows that $C_{j}=e^{2 n \kappa_{j} \pi i}, C_{l}=0(l \neq j)$. Hence, by (5.3), we have

$$
\varphi_{\mu, j}\left(e^{2 n \pi i} t\right)=e^{2 n \kappa_{j} \pi i} \varphi_{\mu, j}(t),
$$

which implies that $\varphi_{*}(\tau)=\tau^{-n \kappa_{j}} \varphi_{\mu, j}\left(\tau^{n}\right)$ satisfies $\varphi_{*}\left(e^{2 \pi i} \tau\right)=\varphi_{*}(\tau)$, and that

$$
\varphi_{*}(\tau) \sim \tau^{-n \kappa_{j}} W_{j}\left(\tau^{n}\right)=Y_{j}\left(\tau^{n}\right) \exp \left(\omega^{j} V_{\infty}\left(\tau^{n}\right)\right)
$$

around $\tau=\infty$. Therefore $Y_{j}(t)$ converges around $t=\infty$. This completes the proof.

Remark 5.1. In the case where $p \in \mathbf{N}$, we call a solution $\chi^{(0)}(t)$ of $(\mathrm{aE})$ a zero-ample solution at $t=0$, if $\chi^{(0)}(1 / \tau)$ is zero-ample at $\tau=\infty$. By Propositions 5.1 and 4.4 , there exists a sector $S_{\mu_{0}}^{0}$ such that

$$
\sharp\left\{t \in S_{\mu_{0}}^{0}\left|\chi^{(0)}(t)=0,1 / r<\right| t \mid<\varepsilon_{0}\right\} \gg r^{p / n}
$$

as $r \rightarrow \infty$. Furthermore, by Propositions 4.4, 5.2 and 5.3, we have the following:

$(1)$ if $\psi^{(0)}(t)(\not \equiv 0)$ is not zero-ample at $t=0$, then, for every $\mu \in \mathbf{Z}$,

$$
\psi^{(0)}(t)=\gamma_{0}^{0} \varphi_{\mu, j_{* *}}^{(0)}(t)
$$


in $S_{\mu}^{0}$, where $\gamma_{0}^{0}(\neq 0)$ and the index $j_{* *}$ are independent of $\mu$;

(2) for each $(\mu, j)$, the solution $\varphi_{\mu, j}^{(0)}(t)$ is not zero-ample at $t=0$, if and only if $Y_{j}^{(0)}(t)$ is convergent around $t=0$.

Remark 5.2. Suppose that $p \leq 0$. Then $t=0$ is at most a regular singular point of $(\mathrm{aE})$. For convenience' sake, in this case, we regard an arbitrary solution $\chi^{(0)}(t)(\not \equiv 0)$ of $(\mathrm{aE})$ as non-zero-ample at $t=0$.

\section{§6. Proofs of Theorems 2.1 and 2.3}

\subsection{Solution of (aE) which is zero-ample neither at $\infty$ nor at 0}

Suppose that (aE) possesses a meromorphic solution $\Phi(t)(\not \equiv 0)$ which is zero-ample neither at $t=\infty$ nor at $t=0$. Then, by Propositions $4.1,5.2$ and 5.3 , around $t=\infty$,

$$
\begin{aligned}
& \Phi(t)=Y^{(\infty)}(t) t^{\kappa(\infty)} \exp \left(\omega_{\infty} V_{\infty}(t)\right), \\
& \kappa(\infty)= \begin{cases}-(n-1) q /(2 n)-R_{n-1}(\infty) / n & \text { if } q / n \notin \mathbf{N}, \\
-(n-1) q /(2 n)-R_{n-1}(\infty) / n+\omega_{\infty} \alpha_{q / n} & \text { if } q / n \in \mathbf{N},\end{cases}
\end{aligned}
$$

in which $Y^{(\infty)}(t)=O[1]_{-1 / n}($ if $q / n \notin \mathbf{N}),=O[1]_{-1}$ (if $\left.q / n \in \mathbf{N}\right)$ satisfies $Y^{(\infty)}(\infty) \neq 0$ and converges near $t=\infty$, and $\omega_{\infty}$ is an $n$-th root of 1 . Furthermore, when $p \geq 1$, by Proposition 4.3 and Remark 5.1, around $t=0$,

$$
\begin{aligned}
& \Phi(t)=Y^{(0)}(t) t^{\kappa(0)} \exp \left(\omega_{0} V_{0}(t)\right), \\
& \kappa(0)= \begin{cases}(n-1) p /(2 n)-R_{n-1}(0) / n & \text { if } p / n \notin \mathbf{N}, \\
(n-1) p /(2 n)-R_{n-1}(0) / n-\omega_{0} \beta_{p / n} & \text { if } p / n \in \mathbf{N},\end{cases}
\end{aligned}
$$

in which $Y^{(0)}(t)=O[1]_{1 / n}$ (if $p / n \notin \mathbf{N}$ ), $=O[1]_{1}$ (if $p / n \in \mathbf{N}$ ) satisfies $Y^{(0)}(0) \neq 0$ and converges near $t=0$, and $\omega_{0}$ is an $n$-th root of 1 . There exists an integer $\iota_{0} \in \mathbf{N}$ such that the multiplicity of every pole of $\Phi(t)$ in $\mathcal{R}$ does not exceed $\iota_{0}$. This fact is verified by substituting a Laurent series expansion of $\Phi(t)$ into (aE) around each pole. We can choose nonnegative integers $\iota(\xi)(\xi \in \mathcal{P})$ as small as possible in such a way that $\Phi(t) \prod_{\xi \in \mathcal{P}}(t-\xi)^{\iota(\xi)}$ is analytic on $\mathcal{R}$. 


\subsubsection{Case $p \geq 1$}

Consider the function

$$
F(t)=\Phi(t)\left(\prod_{\xi \in \mathcal{P}}(t-\xi)^{\iota(\xi)}\right) t^{-\kappa(0)} \exp \left(-\omega_{0} V_{0}(t)-\omega_{\infty} V_{\infty}(t)\right)
$$

which is analytic on $\mathcal{R}$. Then we have

$$
\begin{aligned}
F(t) & =Y^{(0)}(t)\left(\prod_{\xi \in \mathcal{P}}(t-\xi)^{\iota(\xi)}\right) \exp \left(-\omega_{\infty} V_{\infty}(t)\right) \\
& =Y^{(0)}(0) \prod_{\xi \in \mathcal{P}}(-\xi)^{\iota(\xi)}+O\left[t^{1 / n}\right]_{1 / n}
\end{aligned}
$$

near $t=0$, and

$$
\begin{aligned}
F(t) & =Y^{(\infty)}(t)\left(\prod_{\xi \in \mathcal{P}}(t-\xi)^{\iota(\xi)}\right) t^{\kappa(\infty)-\kappa(0)} \exp \left(-\omega_{0} V_{0}(t)\right) \\
& =Y^{(\infty)}(\infty) t^{\kappa^{*}}\left(1+O\left[t^{-1 / n}\right]_{-1 / n}\right), \\
\kappa^{*} & =I(\Phi, \mathcal{P})+\kappa(\infty)-\kappa(0), \quad I(\Phi, \mathcal{P})=\sum_{\xi \in \mathcal{P}} \iota(\xi),
\end{aligned}
$$

near $t=\infty$. By $(6.4), F\left(\tau^{n}\right)$ is entire with respect to $\tau$ and satisfies $F(0) \neq 0$. Hence, by $(6.5), F\left(\tau^{n}\right)=P(\tau)$ is a polynomial in $\tau$, and $n \kappa^{*}=n(I(\Phi, \mathcal{P})+\kappa(\infty)-\kappa(0)) \in \mathbf{N} \cup\{0\}$. This implies that $\Phi(t)$ is written in the form (2.3) with $\kappa=\kappa(0)$. By the definition of $\iota(\xi), P(\tau)$ is not divisible by $\tau^{n}-\xi$ for every $\xi \in \mathcal{P}$ satisfying $\iota(\xi) \in \mathbf{N}$. In particular, if $p / n \in \mathbf{N}$, then we see that $F(t)$ is a polynomial in $t$, and that $\kappa^{*}=I(\Phi, \mathcal{P})+\kappa(\infty)-\kappa(0) \in \mathbf{N} \cup\{0\}$. Suppose that $q / n \notin \mathbf{N}, p / n \in \mathbf{N}$. Then, by (6.2), $\Psi(t)=\Phi(t) t^{-\kappa(0)}$ is single-valued on $\mathbf{C}-\{0\}$. On the other hand, around $t=\infty$,

$$
\Psi(t)=Y^{(\infty)}(t) t^{-\kappa(0)+\kappa(\infty)} \exp \left(\left(\omega_{\infty} n \alpha_{0} / q\right) t^{q / n}\left(1+O\left(t^{-1}\right)\right)\right) .
$$

Since $q / n \notin \mathbf{N}, \Psi(t)$ is not single-valued around $t=\infty$, which is a contradiction. In a similar way, we can show that $q / n \in \mathbf{N}$ and $p / n \notin \mathbf{N}$ do not hold simultaneously. Thus we have proved that either of the following cases occurs:

(a) $q / n \notin \mathbf{N}, p / n \notin \mathbf{N}, p \geq 1,-(n-1)(q+p) / 2-\left(R_{n-1}(\infty)-R_{n-1}(0)\right)+$ $n I(\Phi, \mathcal{P}) \in \mathbf{N} \cup\{0\}$

(b) $q / n \in \mathbf{N}, p / n \in \mathbf{N}, p \geq 1,-(n-1)(q+p) /(2 n)-\left(R_{n-1}(\infty)-\right.$ $\left.R_{n-1}(0)\right) / n+\omega_{\infty} \alpha_{q / n}+\omega_{0} \beta_{p / n}+I(\Phi, \mathcal{P}) \in \mathbf{N} \cup\{0\}$. 
6.1.2. Case $p \leq 0$

We put

$$
G(t)=\Phi(t)\left(\prod_{\xi \in \mathcal{P}}(t-\xi)^{\iota(\xi)}\right) t^{-\kappa(\infty)} \exp \left(-\omega_{\infty} V_{\infty}(t)\right) .
$$

Then $G(t)$ is analytic on $\mathcal{R}$. Consider the case where $q / n \notin \mathbf{N}$. Since

$$
G(t)=Y^{(\infty)}(t) \prod_{\xi \in \mathcal{P}}(t-\xi)^{\iota(\xi)}=t^{I(\Phi, \mathcal{P})} O[1]_{-1 / n}
$$

converges near $t=\infty$, the function $G\left(\tau^{n}\right)$ is analytic on $\mathbf{C}-\{0\}$, and $\tau=\infty$ is at most a pole of $G\left(\tau^{n}\right)$. Hence, observing that $t=0$ is a regular singular point, we have

$$
\begin{aligned}
t^{-\kappa(\infty)} \Phi(t) & =G(t)\left(\prod_{\xi \in \mathcal{P}}(t-\xi)^{-\iota(\xi)}\right) \exp \left(\omega_{\infty} V_{\infty}(t)\right) \\
& =t^{m / n}\left(c_{0}+O[t]_{1 / n}\right), \quad c_{0} \neq 0
\end{aligned}
$$

near $t=0$ for some $m \in \mathbf{Z}$. This implies that, for the solution $\Phi(t)$, $\rho_{m}=m / n+\kappa(\infty)=(2 m-(n-1) q) /(2 n)-R_{n-1}(\infty) / n$ is a characteristic exponent at $t=0$, and hence $\rho_{m}$ is a root of (2.4). By (6.7), $t^{-m / n} G(t)=$ $P\left(t^{1 / n}\right)=O[1]_{1 / n}, P(0) \neq 0$, and hence $P(\tau)$ is a polynomial in $\tau$. Furthermore, by (6.6), $m / n \leq I(\Phi, \mathcal{P})$. When $q / n \in \mathbf{N}$, the function $G(t)$ is analytic on $\mathbf{C}-\{0\}$, and $t=\infty$ is at most a pole of $G(t)$. By an analogous argument, we verify that, for some $m \in \mathbf{Z}$ satisfying $m \leq I(\Phi, \mathcal{P})$, $\tilde{\rho}_{m}=m+\kappa(\infty)=m-(n-1) q /(2 n)-R_{n-1}(\infty) / n+\omega_{\infty} \alpha_{q / n}$ is a root of (2.4), and that $t^{-m} G(t)$ is a polynomial in $t$.

Summing up the facts above, we have the following:

Proposition 6.1. Suppose that there exists a meromorphic solution $\Phi(t)(\not \equiv 0)$ of $(\mathrm{aE})$ which is zero-ample neither at $t=\infty$ nor at $t=0$. Then $\Phi(t)$ is expressible in the form (2.3), and one of the cases (i), (ii), (iii), (iv) of Theorem 2.1 with $I(\Phi, \mathcal{P})$ in place of $I_{\phi}(\mathcal{P})$ occurs.

\subsection{Proof of Theorem 2.1}

Concerning the zero-density we have the following:

Lemma 6.2. Let $\phi(z)=\Phi\left(e^{z}\right)$ be a meromorphic solution of $(\mathrm{E})$. If $\Phi(t)$ is zero-ample at $t=\infty$, then $N(r, 1 / \phi) \gg r^{-1} e^{(q / n) r}$. If $p \geq 1$, and if $\Phi(t)$ is zero-ample at $t=0$, then $N(r, 1 / \phi) \gg r^{-1} e^{(p / n) r}$. 
Proof. Suppose that $\Phi(t)$ is zero-ample at $t=\infty$. Let $S_{\mu}$ be a sector such that Proposition 5.1 is valid for $\chi(t)=\Phi(t)$. Note that, by $t=e^{z}$, the strip $\log M_{\infty}<\operatorname{Re} z<r^{\prime},(n / q)(\mu \pi-\delta)<\operatorname{Im} z<(n / q)(\mu+1) \pi$ is conformally mapped onto the region $\left\{t \in S_{\mu}\left|M_{\infty}<\right| t \mid<e^{r^{\prime}}\right\}$. By Proposition 5.1 the number of zeros of $\phi(z)=\Phi\left(e^{z}\right)$ in $|z|<r$ is estimated as $n(r, 1 / \phi) \gg e^{(q / n) r+O(1 / r)}$, so that

$$
N(r, 1 / \phi) \gg \int_{1}^{r} \frac{1}{\sigma}(n(\sigma, 1 / \phi)-n(0,1 / \phi)) d \sigma \gg r^{-1} e^{(q / n) r} .
$$

The second assertion is verified in a similar way.

Suppose that $\phi(z)=\Phi\left(e^{z}\right)(\not \equiv 0)$ is a meromorphic solution of $(\mathrm{E})$ satisfying $\lambda(\phi)<+\infty$. Then, by Lemma $6.2, \Phi(t)$ is zero-ample neither at $t=\infty$ nor at $t=0$. Combining this fact with Proposition 6.1, we obtain Theorem 2.1 .

\subsection{Proof of Theorem 2.3}

Suppose that a meromorphic solution $\phi(z)=\Phi\left(e^{z}\right)$ of $(\mathrm{E})$ satisfies $\lambda(\phi)=+\infty$. Then, $\Phi(t)$ is zero-ample at $t=0$ or $t=\infty$; otherwise, by Proposition 6.1, we have $\lambda(\phi)<+\infty$. For example consider the case where $\Phi(t)$ is zero-ample at $t=\infty$. Then, by Lemma $6.2, N(r, 1 / \phi) \gg r^{-1} e^{(q / n) r}$. The other cases are treated in a similar way. Thus we obtain (2.7).

Take a polynomial of the form $\Pi(t)=\prod_{\xi \in \mathcal{P}}(t-\xi)^{\delta(\xi)}, \delta(\xi) \in \mathbf{N} \cup\{0\}$ in such a way that $\eta(z)=\phi(z) \Pi\left(e^{z}\right)=\Phi\left(e^{z}\right) \Pi\left(e^{z}\right)$ is entire. It is easy to see that $\eta(z)$ satisfies an equation of the form

$$
\eta^{(n)}+Q_{n-1}\left(e^{z}\right) \eta^{(n-1)}+\cdots+Q_{1}\left(e^{z}\right) \eta^{\prime}+Q_{0}\left(e^{z}\right) \eta=0 .
$$

Here $Q_{h}(t)(h=0,1, \ldots, n-1)$ are rational functions of $t$ whose poles belong to $\mathcal{P}$. All the poles of the coefficients of $\left(\mathrm{E}^{\prime}\right)$ are written in the form $\zeta_{d, l}=z_{d}+2 l \pi i, e^{z_{d}} \in \mathcal{P}\left(d=1, \ldots, d_{0} \leq \sharp \mathcal{P}, l \in \mathbf{Z}\right)$. Consider the domain

$$
\Delta=\mathbf{C}-\bigcup_{d=1}^{d_{0}} \bigcup_{l \in \mathbf{Z}}\left\{z|| z-\zeta_{d, l} \mid \leq(|l|+1)^{-2}\right\} .
$$

All the radiuses of the circles $\Gamma_{r}:|z|=r$ satisfying $\Gamma_{r} \not \subset \Delta$ constitute the set $E_{0} \subset \mathbf{R}_{+}=\{r \mid r>0\}$ of finite linear measure. If $r \in \mathbf{R}_{+}-E_{0}$, then $\Gamma_{r} \subset \Delta$. Note that $|l| \ll \zeta_{d, l}$ as $|l| \rightarrow \infty$. Hence

$$
\log \left|Q_{h}\left(e^{z}\right)\right| \ll r, \quad h=0,1, \ldots, n-1,
$$


as $r \rightarrow \infty, r \notin E_{0}$. For the entire function $\eta(z)=\sum_{k \geq 0} c_{k} z^{k}$, we put

$$
\mu(r, \eta)=\max \left\{\left|c_{k}\right| r^{k} \mid k \geq 0\right\}, \quad \nu(r, \eta)=\max \left\{k|\mu(r, \eta)=| c_{k} \mid r^{k}\right\} .
$$

Then, by the Wiman-Valiron theory ([20], [21]),

$$
\eta^{(h)}(z)=\left(\frac{\nu(r, \eta)}{z}\right)^{h}(1+o(1)) \eta(z), \quad r=|z|, \quad h=1, \ldots, n
$$

for $z$ satisfying $|\eta(z)|=M(r, \eta)=\max \{|\eta(\zeta)||| \zeta \mid=r\},|z| \notin E_{1}$, where $E_{1}$ is a set of finite logarithmic measure. Substituting (6.9) into ( $\left.\mathrm{E}^{\prime}\right)$, and using (6.8), we have $\log \nu(r, \eta) \ll r$ as $r \rightarrow \infty, r \notin F=E_{0} \cup E_{1}$. By [20, Satz 4.4],

$$
\log M(r, \eta) \leq \log (\mu(r, \eta)(\nu(2 r, \eta)+2)) \ll \nu(r, \eta) \log r+\log \nu(2 r, \eta)
$$

as $r \rightarrow \infty$, and hence $\log T(r, \eta) \ll r$, as $r \rightarrow \infty, r \notin F$. Note that $\int_{F} d x / x=u_{0}<+\infty$, and that, for every $r>0, \int_{r}^{U_{0} r} d x / x=2 u_{0}\left(U_{0}=\right.$ $\left.\exp \left(2 u_{0}\right)\right)$. There exists $r^{\prime}=r^{\prime}(r)$ satisfying $r<r^{\prime}<U_{0} r$ and $r^{\prime} \notin F$. Observing that $\log T(r, \eta)$ is monotone increasing, we have $\log T(r, \eta) \leq$ $\log T\left(r^{\prime}, \eta\right) \ll r^{\prime}<U_{0} r$ for $r \geq r_{0}$, where $r_{0}$ is a sufficiently large positive constant. Therefore

$$
\log N(r, 1 / \phi) \ll \log T(r, \eta)+\log T\left(r, \Pi\left(e^{z}\right)\right) \ll r
$$

for $r \geq r_{0}$, which implies (2.6). This completes the proof.

\section{§7. Proof of Theorem 2.2}

By definition, $n_{0}=n / d_{0}$ and $q_{0}=q / d_{0}$ are relatively prime. Since $q / n \notin \mathbf{N}$, we have $d_{0}<n$, so that $n_{0}>1$. By the change of the variable $e^{z / n_{0}}=s$, equation (E) is transformed into

$\left(\mathrm{aE}^{*}\right)$

$$
\check{\vartheta}^{n} w+n_{0} R_{n-1}\left(s^{n_{0}}\right) \check{\vartheta}^{n-1} w+\cdots+n_{0}^{n-1} R_{1}\left(s^{n_{0}}\right) \check{\vartheta} w+n_{0}^{n} R_{0}\left(s^{n_{0}}\right) w=0,
$$

$\check{\vartheta}=s(d / d s)$, where

$$
n_{0}^{n} R_{0}\left(s^{n_{0}}\right)=n_{0}^{n}\left(a_{0} s^{q n_{0}}+a_{1} s^{(q-1) n_{0}}+\cdots+a_{k} s^{(q-k) n_{0}}+\cdots\right) .
$$

Observing that $q n_{0} / n=q_{0} \in \mathbf{N}$, we write

$$
\left[-n_{0}^{n} s^{q n_{0}} \sum_{0 \leq n_{0} k \leq q_{0}} a_{k} s^{-n_{0} k}\right]^{1 / n}=s^{q_{0}} \sum_{k=0}^{\infty} \tilde{a}_{k} s^{-n_{0} k}=s^{q_{0}} \sum_{l=0}^{\infty} A_{l} s^{-l} .
$$


Since $q_{0} / n_{0} \notin \mathbf{N}$

$$
A_{q n_{0} / n}=A_{q_{0}}=0
$$

Suppose that $(\mathrm{E})$ possesses a meromorphic solution $\phi(z)=\Phi\left(e^{z}\right)(\not \equiv 0)$ satisfying $\lambda(\phi)<+\infty$, where $\Phi(t)$ is a meromorphic solution of (aE). Note that $\tilde{\phi}(\zeta)=\phi\left(n_{0} \zeta\right)=\Phi_{0}\left(e^{\zeta}\right)\left(\zeta=z / n_{0}\right)$ also satisfies $\lambda(\tilde{\phi})<+\infty$ as a function of $\zeta$, where $\Phi_{0}(s)$ is a solution of $\left(\mathrm{aE}^{*}\right)$. Then, by Theorem 2.1 with $q n_{0}$ (instead of $q$ ), $\Phi_{0}(s)$ is written in the form

$$
\begin{aligned}
\Phi_{0}(s) & =\left(\prod_{\xi^{\prime} \in \mathcal{P}^{\prime}}\left(s-\xi^{\prime}\right)^{-\iota^{\prime}\left(\xi^{\prime}\right)}\right) P_{*}(s) s^{\kappa_{0}} \exp \left(\omega_{\infty} V_{\infty}^{*}(s)\right), \\
V_{\infty}^{*}(s) & =s^{q_{0}} \sum_{0 \leq l<q_{0}} \frac{A_{l}}{q_{0}-l} s^{-l},
\end{aligned}
$$

where $\omega_{\infty}$ is some $n$-th root of $1, \mathcal{P}^{\prime}=\left\{\xi^{\prime} \in \mathbf{C}-\{0\} \mid \xi^{\prime n_{0}} \in \mathcal{P}\right\}$, and $\iota^{\prime}\left(\xi^{\prime}\right) \in \mathbf{N} \cup\{0\}$. Note that case (iv) of Theorem 2.1 with $q n_{0}$ occurs. For some $m_{0} \in \mathbf{Z}$ satisfying $m_{0} \leq I_{\phi}^{\prime}\left(\mathcal{P}^{\prime}\right)=\sum_{\xi^{\prime} \in \mathcal{P}^{\prime}} \iota^{\prime}\left(\xi^{\prime}\right)$,

$$
\begin{aligned}
\kappa_{0} & =m_{0}-(n-1) q n_{0} /(2 n)-R_{n-1}(\infty) n_{0} / n \\
& =m_{0}-(n-1) q_{0} / 2-R_{n-1}(\infty) / d_{0}
\end{aligned}
$$

(cf. (7.1)), and $P_{*}(s)$ is a polynomial in $s$ satisfying $P_{*}(0) \neq 0$ and $P_{*}\left(\xi^{\prime}\right) \neq 0$ for every $\xi^{\prime} \in \mathcal{P}^{\prime}$ such that $\iota^{\prime}\left(\xi^{\prime}\right) \in \mathbf{N}$. Observing that $\Phi_{0}(s)=\Phi\left(s^{n_{0}}\right)$ (cf. (2.3)), and that, for each $\xi \in \mathcal{P}$,

$$
\left(s^{n_{0}}-\xi\right)^{-\iota(\xi)}=\prod_{j=0}^{n_{0}-1}\left(s-\tilde{\omega}^{j} \xi^{1 / n_{0}}\right)^{-\iota(\xi)}, \quad \tilde{\omega}=\exp \left(2 \pi i / n_{0}\right),
$$

we have $\iota(\xi) \geq \iota^{\prime}\left(\xi^{\prime}\right)$, if $\xi^{\prime n_{0}}=\xi, \xi^{\prime} \in \mathcal{P}^{\prime}$. Hence $m_{0} \leq I_{\phi}^{\prime}\left(\mathcal{P}^{\prime}\right) \leq n_{0} I_{\phi}(\mathcal{P})$. By Theorem 2.1, (iv) (with (c)), $\kappa_{0}$ is a characteristic exponent of $\Phi_{0}(s)$ at $s=0$, and $\Phi_{0}(s)$ is expressed as

$$
\Phi_{0}(s)=s^{\kappa_{0}} \sum_{k \geq 0} c_{0}(k) s^{k}, \quad c_{0}(0) \neq 0
$$

near $s=0$. To derive other characteristic exponents, we note the fact that equation $\left(\mathrm{aE}^{*}\right)$ remains invariant under the replacement of $s$ by $\tilde{\omega}^{j} s$ $(j \in \mathbf{Z})$. Hence, $\Phi_{0}\left(\tilde{\omega}^{j} s\right)\left(j=0,1, \ldots, n_{0}-1\right)$ are solutions of $\left(\mathrm{aE}^{*}\right)$. 
Furthermore these solutions are linearly independent, because the leading terms of $V_{\infty}^{*}\left(\tilde{\omega}^{j} s\right)$ and $V_{\infty}^{*}\left(\tilde{\omega}^{h} s\right)$ coincide with each other, only when $(h-j) q_{0} / n_{0} \in \mathbf{Z}$, which is equivalent to $(h-j) / n_{0} \in \mathbf{Z}$. It is easy to see that $\phi_{j}(z)=\phi(z+2 j \pi i)=\Phi_{0}\left(e^{(z+2 j \pi i) / n_{0}}\right)\left(j=0,1, \ldots, n_{0}-1\right)$ satisfy $\lambda\left(\phi_{j}\right)<+\infty$. By $(7.3)$, we have, for $j=0,1, \ldots, n_{0}-1$,

$$
\psi_{j}=\Phi_{0}\left(\tilde{\omega}^{j} s\right)=s^{\kappa_{0}} \sum_{k \geq 0} c_{j}(k) s^{k}, \quad c_{j}(0)=\tilde{\omega}^{\kappa_{0} j} c_{0}(0) \neq 0 .
$$

From these solutions, we derive the linearly independent solutions

$$
\begin{array}{ll}
\psi_{0}=s^{\kappa_{0}}\left(c_{0}(0)+O(s)\right), & \\
\psi_{j}-\tilde{\omega}^{\kappa_{0} j} \psi_{0}=s^{\kappa_{0}+l(j)}\left(c_{j}^{1}(0)+O(s)\right), & l(j) \in \mathbf{N}, \quad c_{j}^{1}(0) \neq 0, \\
& 1 \leq j \leq n_{0}-1 .
\end{array}
$$

Thus we obtain the sequence

$$
\kappa_{0}<\kappa_{1}=\kappa_{0}+l\left(j_{1}\right) \leq \kappa_{0}+l\left(j_{2}\right) \leq \cdots \leq \kappa_{0}+l\left(j_{n_{0}-1}\right)
$$

which contains at least two distinct characteristic exponents $\kappa_{0}, \kappa_{1}$. Repeating this procedure within $\left(n_{0}-1\right)$ times, we obtain $n_{0}$ distinct characteristic exponents $\kappa_{j}=\kappa_{0}+l_{j}\left(0 \leq j \leq n_{0}-1\right), l_{j} \in \mathbf{Z}, l_{0}=0<l_{1}<\cdots<l_{n_{0}-1}$. Hence they satisfy equation (2.5). This completes the proof.

\section{§8. Proof of Theorem 2.4}

Suppose that there exist linearly independent entire solutions $\chi_{j}(z)=$ $\Phi_{j}\left(e^{z}\right)(j=0,1, \ldots, n-1)$ satisfying $\lambda\left(\chi_{j}\right)<+\infty$.

\subsection{Case $p \geq 1$}

Under the assumptions $\mathcal{P}=\emptyset, R_{n-1}(t) \equiv 0$, the case (i) of Theorem 2.1 does not occur. It is sufficient to treat the case where $q / n \in \mathbf{N}, p / n \in \mathbf{N}$. Then, each $\Phi_{j}(t)$ is zero-ample neither at $t=\infty$ nor at $t=0$. Using Propositions 4.1, 4.3, 5.2 and 5.3, we have, for $j=0,1, \ldots, n-1$,

$$
\Phi_{j}(t)=t^{\kappa_{j}} Y_{j}^{(\infty)}(t) \exp \left(\omega^{j} V_{\infty}(t)\right), \quad \omega=\exp (2 \pi i / n)
$$

(the indices of $\Phi_{0}(t), \ldots, \Phi_{n-1}(t)$ are suitably rearranged if necessary), and

$$
\Phi_{j}(t)=t^{\kappa_{j}(0)} Y_{j}^{(0)}(t) \exp \left(\omega^{\varepsilon(j)} V_{0}(t)\right)
$$


Here $Y_{j}^{(\infty)}(t)=O[1]_{-1}$ and $Y_{j}^{(0)}(t)=O[1]_{1}$ converge near $t=\infty$ and near $t=0$, respectively, $\varepsilon(j)(j=0,1, \ldots, n-1)$ are integers satisfying $0 \leq \varepsilon(j) \leq n-1$, and

$$
\kappa_{j}=-(n-1) q /(2 n)+\omega^{j} \alpha_{q / n}, \quad \kappa_{j}(0)=(n-1) p /(2 n)-\omega^{\varepsilon(j)} \beta_{p / n} .
$$

By the same argument as in the proof of Theorem 2.1 (cf. Section 6.1.1), we have, for $j=0,1, \ldots, n-1$,

$$
(n-1)(p+q)=2 n\left(-m_{j}^{\prime}+\omega^{j} \alpha_{q / n}+\omega^{\varepsilon(j)} \beta_{p / n}\right)
$$

where $m_{j}^{\prime} \in \mathbf{N} \cup\{0\}\left(I_{\chi_{j}}(\mathcal{P})=0\right)$. Note that $\varepsilon(j) \neq \varepsilon\left(j^{\prime}\right)$ for $j \neq j^{\prime}$, because $\Phi_{0}(t), \ldots, \Phi_{n-1}(t)$ are linearly independent solutions of (aE). Summing (8.1.j) over $0 \leq j \leq n-1$, we have $n(n-1)(p+q)=-2 n \sum_{j=0}^{n-1} m_{j}^{\prime} \leq 0$, which is a contradiction.

\subsection{Case $p \leq 0$}

By Theorem 2.1 with $\mathcal{P}=\emptyset$,

$$
\Phi_{j}(t)=t^{\kappa_{j}} P_{j}(t) \exp \left(\omega^{j} V_{\infty}(t)\right), \quad j=0,1, \ldots, n-1,
$$

where $P_{j}(t)(j=0, \ldots, n-1)$ are polynomials in $t$ and

$$
\kappa_{j}=m_{j}^{\prime \prime}-(n-1) q /(2 n)+\omega^{j} \alpha_{q / n}, \quad m_{j}^{\prime \prime} \leq 0
$$

Hence

$$
\sum_{j=0}^{n-1} \kappa_{j}=-(n-1) q / 2+\sum_{j=0}^{n-1} m_{j}^{\prime \prime}<0
$$

On the other hand, by assumption, $\kappa_{j}(0 \leq j \leq n-1)$ are $n$ distinct characteristic exponents at $t=0$. Then, from $(2.4)$, we have $\sum_{j=0}^{n-1} \kappa_{j}=0$, which contradicts (8.2). This completes the proof.

\section{REFERENCES}

[1] A. Baesch, On the explicit determination of certain solutions of periodic differential equations of higher order, Results Math., 29 (1996), 42-55.

[2] A. Baesch and N. Steinmetz, Exceptional solutions of $n$-th order periodic linear differential equations, Complex Variables Theory Appl., 34 (1997), 7-17. 
[3] W. Balser, W. B. Jurkat and D. A. Lutz, Birkhoff invariants and Stokes' multipliers for meromorphic linear differential equations, J. Math. Anal. Appl., 71 (1979), 48-94.

[4] S. B. Bank, Three results in the value-distribution theory of solutions of linear differential equations, Kodai Math. J., 9 (1986), 225-240.

[5] S. B. Bank, On determining the location of complex zeros of solutions of certain linear differential equations, Ann. Mat. Pura Appl. (4), 151 (1988), 67-96.

[6] S. B. Bank, On the oscillation theory of periodic linear differential equations, Appl. Anal., 39 (1990), 95-111.

[7] S. B. Bank, On the explicit determination of certain solutions of periodic differential equations, Complex Variables Theory Appl., 23 (1993), 101-121.

[8] S. B. Bank and I. Laine, Representations of solutions of periodic second order linear differential equations, J. Reine Angew. Math., 344 (1983), 1-21.

[9] S. B. Bank and I. Laine, On the zeros of meromorphic solutions of second order linear differential equations, Comment. Math. Helv., 58 (1983), 656-677.

[10] S. B. Bank, I. Laine and J. K. Langley, On the frequency of zeros of solutions of second order linear differential equations, Results Math., 10 (1986), 8-24.

[11] S. B. Bank and J. K. Langley, Oscillation theory for higher order linear differential equations with entire coefficients, Complex Variables Theory Appl., 16 (1991), 163-175.

[12] S. B. Bank and J. K. Langley, Oscillation theorems for higher order linear differential equations with entire periodic coefficients, Comment. Math. Univ. St. Paul., 41 (1992), 65-85.

[13] Y. M. Chiang and I. Laine, Some oscillation results for linear differential equations in the complex plane, Japan. J. Math., 24 (1998), 367-402.

[14] Y. M. Chiang, I. Laine and S. Wang, An oscillation result of a third order linear differential equation with entire periodic coefficients, Complex Variables Theory Appl., 34 (1997), 25-34.

[15] Y. M. Chiang and S. Wang, Oscillation results on certain higher order linear differential equations with periodic coefficients in the complex plane, J. Math. Anal. Appl., 215 (1997), 560-576.

[16] S. Gao, A further result on the complex oscillation theory of periodic second order linear differential equations, Proc. Edinburgh Math. Soc. (2), 33 (1990), 143-158.

[17] G. G. Gundersen, On the real zeros of solutions of $f^{\prime \prime}+A(z) f=0$ where $A(z)$ is entire, Ann. Acad. Sci. Fenn. Math., 11 (1986), 275-294.

[18] W. K. Hayman, Meromorphic Functions, Clarendon, Oxford, 1964.

[19] S. Hellerstein and J. Rossi, Zeros of meromorphic solutions of second order linear differential equations, Math. Z., 192 (1986), 603-612.

[20] G. Jank and L. Volkmann, Einführung in die Theorie der Ganzen und Meromorphen Funktionen mit Anwendungen auf Differentialgleichungen, Birkhäuser, Basel, Boston, Stuttgart, 1985.

[21] I. Laine, Nevanlinna Theory and Complex Differential Equations, de Gruyter, Berlin, 1993. 
[22] I. Laine and T. Sorvali, Local solutions of $w^{\prime \prime}+A(z) w=0$ and branched polymorphic functions, Results Math., 10 (1986), 107-129.

[23] J. K. Langley, Some oscillation theorems for higher order linear differential equations with entire coefficients of small growth, Results Math., 20 (1991), 517-529.

[24] Y. Sibuya, Simplification of a system of linear ordinary differential equations about a singular point, Funkcial. Ekvac., 4 (1962), 29-56.

[25] Y. Sibuya, Global Theory of a Second Order Linear Ordinary Differential Equation with a Polynomial Coefficient, North-Holland, Amsterdam, 1975.

[26] W. Wasow, Asymptotic Expansions for Ordinary Differential Equations, Interscience, New York, London, Sydney, 1965.

Department of Mathematics

Keio University

3-14-1 Hiyoshi

Kohoku-ku

Yokohama, 223-8522

Japan

shimomur@math.keio.ac.jp 